Research Paper

\title{
Combination of Diane-35 and Metformin to Treat Early Endometrial Carcinoma in PCOS Women with Insulin Resistance
}

\author{
Xin $\mathrm{Li}^{1}{ }^{1}$, Yan-Rong Guo ${ }^{1}$, Jin-Fang Lin ${ }^{1 凶}$, Yi Feng2, 3, Håkan Billig², and Ruijin Shao ${ }^{\circledR}$ \\ 1. Department of Gynecology, Obstetrics and Gynecology Hospital of Fudan University, Shanghai 200011, China \\ 2. Department of Physiology/Endocrinology, Institute of Neuroscience and Physiology, The Sahlgrenska Academy, University of \\ Gothenburg, Gothenburg 40530, Sweden \\ 3. Department of Integrative Medicine and Neurobiology, State Key Lab of Medical Neurobiology, Shanghai Medical College and Institute \\ of Acupuncture Research (WHO Collaborating Center for Traditional Medicine), Institute of Brain Science, Fudan University, Shanghai \\ 200032, China
}

$\square$ Corresponding authors: Ruijin Shao, M.D., Ph.D., Tel: +46 31 7863408; Fax: +46 31 7863512; E-mail: ruijin.shao@fysiologi.gu.se. Jin-Fang Lin, M.D., Tel: +86 15921516258; Fax: +86 21 63450944; E-mail: linjinfang@shmu.edu.cn

(1) Ivyspring International Publisher. This is an open-access article distributed under the terms of the Creative Commons License (http://creativecommons.org/ licenses/by-nc-nd/3.0/). Reproduction is permitted for personal, noncommercial use, provided that the article is in whole, unmodified, and properly cited.

Received: 2013.10.29; Accepted: 2014.01.14; Published: 2014.01.28

\begin{abstract}
Background: Young women with polycystic ovary syndrome (PCOS) have a high risk of developing endometrial carcinoma. There is a need for the development of new medical therapies that can reduce the need for surgical intervention so as to preserve the fertility of these patients. The aim of the study was to describe and discuss cases of PCOS and insulin resistance (IR) women with early endometrial carcinoma while being co-treated with Diane-35 and metformin.

Methods: Five PCOS-IR women who were scheduled for diagnosis and therapy for early endometrial carcinoma were recruited. The hospital records and endometrial pathology reports were reviewed. All patients were co-treated with Diane- 35 and metformin for 6 months to reverse the endometrial carcinoma and preserve their fertility. Before, during, and after treatment, endometrial biopsies and blood samples were obtained and oral glucose tolerance tests were performed. Endometrial pathology was evaluated. Body weight (BW), body mass index (BMI), follicle-stimulating hormone (FSH), luteinizing hormone (LH), total testosterone (TT), sex hormone-binding globulin (SHBG), free androgen index (FAI), insulin area under curve (IAUC), and homeostasis model assessment of insulin resistance (HOMA-IR) were determined.

Results: Clinical stage la, low grade endometrial carcinoma was confirmed before treatment. After 6 months of co-treatment, all patients showed normal epithelia. No evidence of atypical hyperplasia or endometrial carcinoma was found. Co-treatment resulted in significant decreases in BW, BMI, TT, FAI, IAUC, and HOMA-IR in parallel with a significant increase in SHBG. There were no differences in the FSH and $\mathrm{LH}$ levels after co-treatment.

Conclusions: Combined treatment with Diane- 35 and metformin has the potential to revert the endometrial carcinoma into normal endometrial cells in PCOS-IR women. The cellular and molecular mechanisms behind this effect merit further investigation.
\end{abstract}

Key words: PCOS, progesterone resistance, insulin resistance, steroid hormone receptors, endometrial carcinoma

\section{INTRODUCTION}

Women with polycystic ovary syndrome (PCOS) present with hyperandrogenism, chronic oligo/anovulation, polycystic ovaries, obesity, and metabolic disturbances including insulin resistance with consequent compensatory hyperinsulinemia [1, 2]. Approximately $4 \%-18 \%$ of women of reproductive 
age worldwide suffer from this disease [3]. While the exact underlying molecular mechanisms behind the development of PCOS remain unclear, the PCOS-related endocrine and metabolic abnormalities have been considered to contribute to endometrial dysfunction-induced infertility and endometrial disorders such as endometrial hyperplasia [4]. It is widely accepted that prolonged estrogen excess or lack of progesterone results in atypical endometrial hyperplasia, which is the precursor of endometrial carcinoma [4,5]. Endometrial cancer is a potentially life-threatening gynecological malignancy [6], and more than $80 \%$ of all endometrial cancers are estrogen-dependent endometrial carcinomas [7]. Young women with PCOS have a high risk of developing endometrial carcinoma [8], and it has been reported that PCOS women with endometrial hyperplasia have a four times greater risk of developing endometrial carcinoma than non-PCOS women [9]. Studies have shown that changes in endogenous hormone metabolism, insulin resistance (IR), and obesity can all contribute to the development of endometrial cancer [10, $11]$.

Surgical procedures, including total hysterectomy and bilateral salpingo-oophorectomy, are still the first line and most effective treatments for early stage endometrial cancer although this procedure does not target endometrial carcinoma-specific pathways [7]. Because young women with PCOS usually wish to retain their fertility potential, a challenge to the research community has been to develop new non-surgical and conservative treatments for endometrial carcinoma [12]. We and others have previously reported that the combination of metformin ( $N, N$-dimethylbiguanide) and oral contraceptives are sufficient to reverse atypical endometrial hyperplasia in PCOS-IR women and preserve their fertility [13, 14]. In this report, we present the first pathological findings from five PCOS-IR women with confirmed early endometrial carcinoma, and correlate these findings with clinical and laboratory data in these patients after 6 months of combined treatment with Diane-35 and metformin. The present case report suggests that our non-surgical, interventional treatment successfully reverses early endometrial carcinoma in PCOS-IR women.

\section{METHODS and CASE PRESENTATION}

\section{Patients, Treatments, and Methods}

From April 2011 to February 2013, five childbearing aged women (age 26-32 years; mean, 29.0 years) were recruited and diagnosed with PCOS according to the Rotterdam criteria provided by the American Society for Reproductive Medicine and the
European Society for Human Reproduction and Embryology [15]. A diagnosis of PCOS was made if at least two of the following criteria were met: 1) oligo/anovulation, 2) signs of hyperandrogenism (i.e., hirsutism and acne) and/or biochemical measurements (Table 1), or 3) enhanced ovaries (at least 12 discrete follicles of 2-9 $\mathrm{mm}$ in diameter in one ovary or the ovarian volume $>10 \mathrm{~cm}^{3}$ observed by transvaginal ultrasonography). Women with other androgen-excess disorders or specific etiologies including congenital adrenal hyperplasia, Cushing's syndrome, thyroid hormone abnormalities, hyperprolactinemia, or ovarian/adrenal tumors were excluded. All patients had no history of previous first-trimester miscarriage or pregnancy. Three patients with endometrial carcinoma had been treated orally with megestrol progesterone (160 mg daily), a potent agonist of the progesterone receptor, for three months and progesterone resistance in these patients was confirmed before our combination treatment. None of the women had received metformin or letrozole, an aromatase inhibitor, previously. The local ethics board approved the treatment protocol, and written informed consent to the therapeutic procedure was obtained from all patients.

Patient 1 presented with oligomenorrhea and underwent transvaginal ultrasound scan followed by endometrial curettage and subsequent hysteroscopy. Patient 2 presented with anemia and menorrhagia and underwent endometrial curettage. Patients 3-5 presented with irregular vaginal bleeding and underwent endometrial curettage. Patient 3 with type II diabetes was also treated with pioglitazone $(30 \mathrm{mg}$ daily) during the treatment period. Hysteroscopy was performed in all patients before and after combined treatment with Diane-35 and metformin, and these women showed endometrial polypoid or papillary masses and normal endometrium that are associated with vascular proliferation. In contrast, there were no obvious signs of endometrial abnormalities in any of the patients after combined treatment. The pathological and ultrasonography diagnoses confirmed that the lesions and abnormalities were limited to the endometrial layer. If myometrial invasion was suspected, the patients were examined and confirmed by further MR scans.

Measurement of endometrial thickness by transvaginal ultrasonography was performed by the same investigator in all patients. The endometrial thickness was $6-11 \mathrm{~mm}$ before combined treatment and $5-8 \mathrm{~mm}$ on the $5^{\text {th }}$ day of the medication-induced withdrawal bleeding after combined treatment. Diane- 35 contains $2 \mathrm{mg}$ of cyproterone acetate plus 35 $\mu \mathrm{g}$ of ethinyl estradiol per dose and is used for reducing androgen levels and restoring regular menses 
[16]. Two patients whose endometrial thickness was less than $6 \mathrm{~mm}$ were given oral doses of Diane-35 (daily, Bayer Schering Pharma AG, Germany) in a repeated cycle of 21 days/month with medication followed by 7 days without medication. The transvaginal ultrasound scans showed that the other three patients had an endometrial thickness of more than 7 $\mathrm{mm}$, and these women were treated orally with medroxyprogesterone acetate (10 $\mathrm{mg}$ daily), a potent agonist of the progesterone/androgen receptor, for 10 days to induce uterine withdrawal bleeding. On day 5, these three patients were co-treated with Diane-35 the same as other two patients. Metformin $(1000 \mathrm{mg}$, Sino-American Shanghai Squibb Pharmaceuticals Ltd., China) was given daily.

All patients underwent transvaginal ultrasound on the $5^{\text {th }}$ day of menstrual bleeding to evaluate the endometrial and myometrial conditions of the uterus. After 3 months and after 6 months of treatment, blood samples were obtained on day 2-5 of the uterine withdrawal bleeding during the Diane-35-free period. Liver and kidney function was examined and an endometrial biopsy was obtained either through endometrial curettage or hysteroscopy to evaluate the safety of the treatment. At the end of the 6-month treatment period, all patients were assessed by hysteroscopy 2 weeks after their final medication-induced withdrawal bleeding. Endometrial biopsies in patients before, during, and after treatment were fixed in $4 \%$ phosphate-buffered formaldehyde, embedded in paraffin, cut into sections, and stained with hematoxylin and eosin. Each endometrial sample was diagnosed and graded by routine pathology analysis according to the cancer staging system of the International Federation of Gynecology and Obstetrics [17]. Myometrial invasion was not seen in any of the patients. Cervical ThinPrep cytologic tests were performed, and all patients were negative for cervical cancer and precancerous lesions.

Body weight (BW), body mass index (BMI), follicle-stimulating hormone (FSH), luteinizing hormone $(\mathrm{LH})$, total testosterone (TT), sex hormone-binding globulin (SHBG), free androgen index (FAI), insulin area under curve (IAUC), and homeostasis model assessment of insulin resistance (HOMA-IR) score were determined before and after treatment. The oral glucose tolerance test (OGTT) was performed as previously described [18]. FAI and HOMA-IR were calculated according to the following equations: $\mathrm{FAI}=$ [testosterone $(\mathrm{nmol} / \mathrm{L}) \times 100] /$ SHBG $(\mathrm{nmol} / \mathrm{L})$ [19], and HOMA-IR $=[$ fasting plasma glucose $(\mathrm{mmol} / \mathrm{L}) \times$ fasting plasma insulin (mIU/L)] / 22.5 [20]. IAUC was calculated by the standard trapezoid method. BMI was used for identification of the degree of obesity, and BMI $\geq 25 \mathrm{~kg} / \mathrm{m}^{2}$ was used as the cut-off for obesity according to the World Health Organization 2000 International Year of Obesity Task Force's proposed diagnostic criteria for the Asia-Pacific region.

Patients were advised to control body weight and increase insulin sensitivity through standardized diet and exercise training regimens during treatment. Total daily caloric intake and energy expenditure were calculated based on the patient's height, weight, and daily physical activity intensity. The type of exercise training was individualized and included simple exercises such as fast walking (120-150 steps/minute). To achieve target heart rates (HRs) for durations of 20 to 30 minutes (daily exercise), patients were taught to monitor their HRs, which was calculated as $\mathrm{HR}=[(220-$ age $) \times 0.7]$ [21]. The patients needed to exercise either five days/week or at least every other day/week for 6 months.

\section{Endometrial histopathological findings}

Before treatment, clinical stage 1a, low grade endometrial carcinoma was confirmed in all five PCOS-IR women in this report. The early endometrial carcinoma exhibited a papillary proliferative glandular epithelial pattern (Fig. 1A and B). During co-treatment, we found examples of both complex atypical hyperplasia and complex hyperplasia. Although the epithelial proliferation and irregular pattern of gland growth were found in both types of hyperplasia, cytological atypia was present in complex atypical hyperplasia (Fig. 1C and D) but not in complex hyperplasia (Fig. 1E and F). However, the glands grew in a regular pattern and no complex and/or atypical hyperplasia was observed after the 6-month co-treatment (Fig. 1G and H). Importantly, three of five patients showed improved menstrual cycle regularity and positive ovulatory response, such as the occurrence of ovulation, within 6 months after the 6-month co-treatment.

\section{Hormonal and metabolic parameters}

The average values of BW and BMI decreased significantly in response to the described treatment with the plasma concentrations of TT and FAI (Table 1). Co-treatment did not significantly change the levels of FSH or LH or the LH/FSH ratio. SHBG levels were significantly increased in co-treated patients (Table 1). The OGTT results showed that the mean peak plasma glucose level was similar before and after treatment (Fig. 2A), but the mean peak plasma insulin level was lower after co-treatment than before co-treatment (Fig. 2B). The only difference in plasma glucose levels after co-treatment was a decrease in glucose levels at 3 hours after the start of the OGTT (Fig. 2A). In contrast, plasma insulin levels were significantly decreased after co-treatment at baseline and 
at 2 and 3 hours after the start of the OGTT (Fig. 2B). These OGTT results (Fig. 2B) were analyzed by IAUC, which also showed a significant difference. The fasting plasma levels of glucose and insulin were used to calculate the measure of insulin resistance (HOMA-IR). There was a significant decrease in the HOMA-IR, which indicated a either a significant decrease in IR or an induction of insulin sensitivity (Table 1).
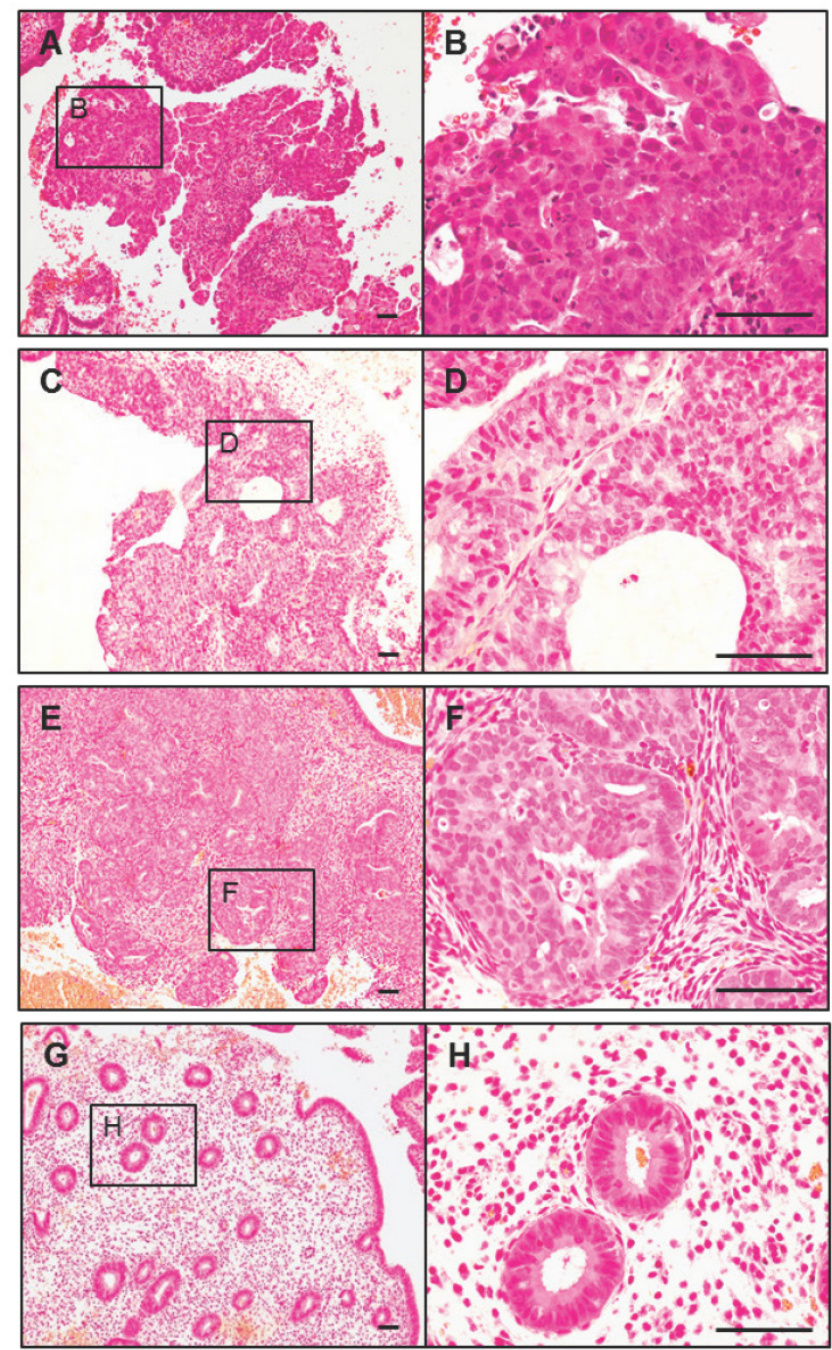

Figure I. Histopathological findings in the endometrium from PCOS-IR women before $(A$ and $B)$, during $(C-F)$, and after $(G$ and $H)$ the 6-month co-treatment with Diane-35 and metformin. Representative microscopic photomicrographs of early endometrial carcinoma (A and $B$, clinical stage Ia, grade I), complex hyperplasia with atypia (C and D), complex hyperplasia ( $E$ and $F)$, and normal endometria ( $G$ and $H)$. Microscopic images stained with hematoxylin and eosin. Scale bar: $100 \mu \mathrm{m}$.
A

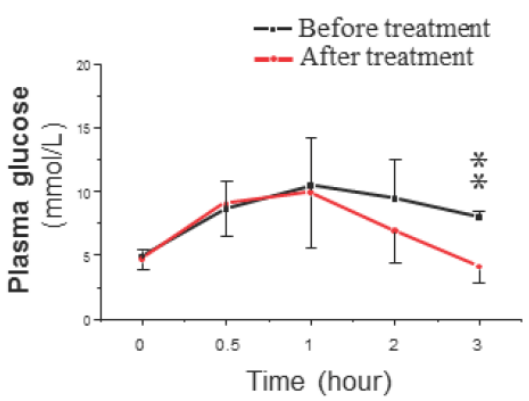

B

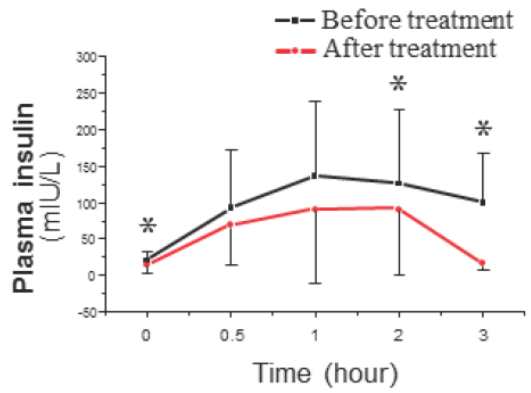

Figure 2. Time course of plasma glucose (A) and insulin (B) concentrations during a 3-hour OGTT in PCOS-IR women before (black line) and after (red line) the 6-month co-treatment with Diane-35 and metformin. All statistical analyses were performed using SPSS version 21.0 for Windows (SPSS Inc., Chicago, IL). Data were analyzed by paired-samples $t$-test and are shown as the mean $\pm \mathrm{SD}$. $*=P<0.05$ and was considered significant.

Table I Relevant clinical, hormonal, and metabolic features of PCOS-related insulin-resistance patients $(n=5)$ before and after a 6-month treatment with Diane-35 and metformin

\begin{tabular}{|c|c|c|c|c|}
\hline & Normal range & $\begin{array}{l}\text { Before } \\
\text { treatment }\end{array}$ & $\begin{array}{l}\text { After } \\
\text { treatment }\end{array}$ & $\begin{array}{l}P \\
\text { value }\end{array}$ \\
\hline BW (kg) & - & $64.40 \pm 2.97$ & $60.20 \pm 3.35$ & 0.003 \\
\hline $\begin{array}{l}\mathrm{BMI} \\
\left(\mathrm{kg} / \mathrm{m}^{2}\right)\end{array}$ & - & $25.86 \pm 0.79$ & $24.16 \pm 0.85$ & 0.004 \\
\hline FSH (IU/L) & $\begin{array}{l}3.3-22.2 \text { (follicular } \\
\text { phase) }\end{array}$ & $5.77 \pm 1.73$ & $3.73 \pm 2.12$ & 0.263 \\
\hline LH (IU/L) & $\begin{array}{l}2.0-12.0 \text { (follicular } \\
\text { phase) }\end{array}$ & $10.00 \pm 5.26$ & $4.50 \pm 2.07$ & 0.068 \\
\hline LH/FSH & - & $1.88 \pm 1.26$ & $1.26 \pm 0.20$ & 0.387 \\
\hline $\begin{array}{l}\mathrm{TT} \\
(\mathrm{nmol} / \mathrm{L})\end{array}$ & $0.52-1.77$ & $2.11 \pm 0.54$ & $1.59 \pm 0.64$ & 0.025 \\
\hline $\begin{array}{l}\text { SHBG } \\
(\mathrm{nmol} / \mathrm{L})\end{array}$ & $19.8-155.2$ & $33.00 \pm 21.28$ & $\begin{array}{l}162.48 \pm \\
94.24\end{array}$ & 0.029 \\
\hline FAI & $0.96-4.62$ & $8.06 \pm 3.58$ & $1.74 \pm 1.79$ & 0.010 \\
\hline $\begin{array}{l}\text { IAUC } \\
(\mathrm{mIU} / \mathrm{L})\end{array}$ & - & $\begin{array}{l}324.26 \pm \\
266.38\end{array}$ & $\begin{array}{l}213.21 \pm \\
198.78\end{array}$ & 0.038 \\
\hline HOMA-IR & - & $1.33 \pm 0.55$ & $0.85 \pm 0.80$ & 0.025 \\
\hline
\end{tabular}

BW, body weight; BMI, body mass index; FSH, follicle-stimulating hormone; $\mathrm{LH}$, luteinizing hormone; TT, total testosterone; $\mathrm{SHBG}$, sex hormone-binding globulin; FAI, free androgen index; IAUC, insulin area under curve; HOMA-IR, homeostasis model assessment of insulin resistance. All statistical analyses were performed using SPSS version 21.0 for Windows (SPSS Inc., Chicago, IL). Data were analyzed by paired-samples $t$-test and were presented as mean \pm SD. $P<0.05$ was considered significant. 


\section{DISCUSSION}

Young women with PCOS commonly develop endometrial hyperplasia and carcinomas [8]. Because surgery is currently the only truly effective means of treating endometrial cancer, there is a need for the development of new medical therapies that can reduce the need for surgical intervention so as to preserve the fertility of these patients [7]. We show that combined treatment of PCOS-IR women with Diane-35 and metformin improves their hyperandrogenemia and IR and reverts their early endometrial carcinoma into normal endometria.

\section{Progesterone resistance in PCOS women}

The human endometrium is a steroid hormone-responsive tissue composed of luminal and glandular epithelial cells, stroma with stromal fibroblastic cells, immunocompetent cells, and blood vessels [22]. The coordinated interaction between estrogen and progesterone is essential for controlling epithelial and stromal cell proliferation, differentiation, secretion, and apoptosis [6]. The coordinated timing of all of these events is necessary to support the key functions of the endometrium, including implantation, pregnancy, menstruation, and endometrial repair [23]. However, the two steroid hormones often act in an opposite manner in the endometrium [6]. It is known that estrogen initiates hypertrophy and hyperplasia of endometrial epithelial cells and that progesterone is involved in the inhibition of estrogen-mediated proliferation [24].

The endometrium from PCOS women is different from normal endometrium, and it experiences sustained and persistent estrogen stimulation but minimal or completely absent progesterone stimulation $[4,23]$. Therefore, progesterone-based oral contraceptives are used for inhibition of endometrial hyperproliferation and improvement of menstrual dysfunction [25]. In fact, approximately $92.5 \%$ of PCOS women who do not develop atypical endometrial hyperplasia after treatment have a return to normal endometria [26]. Furthermore, treatment with megestrol progesterone or medroxyprogesterone can also improve certain cases of endometrial atypical hyperplasia in some PCOS women [27]. However, such therapeutic approaches often lead to disappointing results. There is substantial evidence that approximately $30 \%$ of PCOS women develop progesterone resistance [28], which is defined as the failure to respond to a 3-month, high-dose progesterone treatment regimen [23], and this results in the acceleration of atypical hyperplasia and further transformation to endometrial cancer.

\section{Steroid hormones and their receptor-mediated signaling in PCOS women}

The morphological and physiological alteration of the endometrium is highly regulated by steroid hormone actions [24]. Estrogen and progesterone mediate their endometrial effects through estrogen receptors (ER) and progesterone receptors (PR). ER and PR are coexpressed in human endometrium [23, 29], and an imbalance between the ER- and PR-mediated signaling pathways results in endometrial dysfunction [23, 24]. However, multiple studies have shown that endometrial ER expression is gradually increased in women with PCOS following the onset of endometrial hyperplasia and that endometrial PR expression is decreased in PCOS women with endometrial hyperplasia compared to PCOS women without endometrial hyperplasia [23] (Fig. 3). Similar to the endometrium from PCOS women, endometrial carcinoma contains ER and PR [7], and endometrial defects that lead to progression of endometrial carcinoma have been related to defects in steroid hormone metabolism [11]. Although very little is known about the endometrial expression patterns of ER and PR in PCOS women with endometrial carcinoma, these data suggest that the regulation of ER and PR expression or the activation of ER-and/or PR-mediated pathways is involved in the pathogenesis of endometrial hyperplasia and carcinoma in PCOS women.

Insulin-like growth factor-1 (IGF-1), a key growth factor in the endometrium, is involved in the regulation of cell proliferation and differentiation [30]. Steroid hormones regulate IGF-1 expression and signaling, and the proliferative estrogen has been shown to increase endometrial IGF-1 synthesis in vitro [31]. The anti-proliferative progesterone does not directly regulate endometrial IGF-1 expression and activity. Instead, we and others have shown that progesterone stimulates stromal insulin-like growth factor binding protein-1 (IGFBP-1) expression in vitro $[30,31]$ and this in turn inhibits IGF-1 expression and activity [11]. These data demonstrate that different steroid hormone-mediated regulatory mechanisms affect IGF-1 activity in the endometrium.

IGF-1 can directly stimulate cell proliferation and inhibit apoptosis by binding to the IGF-1 receptor (IGF-1R) that is expressed on the epithelial and stromal cells of the endometrium [30]. Improper IGFBP-1 and IGF-1R expression might contribute to endometrial pathology. For example, IGFBP-1 expression is undetectable or only minimally present in endometrial hyperplasia and cancer [32] whereas IGF-1R expression is elevated in endometrial hyperplasia [33]. Thus, IGF-1 signaling likely plays a significant role in the development of endometrial hyperplasia and the progression of endometrial carcinoma in PCOS 
women, but the exact role that IGF-1 plays in this process has not yet to be determined.

PCOS women have elevated levels of androgens $[1,2]$, but the function of endogenous androgens in the endometrium is not well understood. In addition to androgens, several studies have shown that estrogen can regulate the androgen receptor (AR) in human endometrium [34, 35]. Increased endometrial AR expression in PCOS women during the development of endometrial hyperplasia [23] (Fig. 3) might be due to prolonged exposure to estrogen. Furthermore, treatment with RU 486, a PR antagonist, increases both epithelial and stromal AR expression in human endometrium [36] suggesting that the inhibitory effects of progesterone on AR expression in the endometrium are mediated by PR. In addition, we are aware that surgical procedures are the most effective treatments for the early stages of EC, and analysis of endometrial PR expression in women with EC after hysterectomy is routinely used for the guidance of progestin medication. Because a conservative treatment is selected, it is important to determine the different expression and regulation of endometrial PR in PCOS-IR women with EC before, during, and after co-treatment.

The molecular mechanisms behind the development of endometrial carcinoma in PCOS women are not well understood [28]. Because PCOS women show oligo/anovulation and abnormal menstrual cycle regularity $[1,2]$, it is tempting to speculate that the endometrial stromal cells lack the progesterone-dependent and PR-mediated synthesis and secretion of IGFBP-1. A lack of IGFBP-1 would release the inhibition of IGF-1, and subsequent IGF-1 signaling in the epithelial and stromal cells of the endometrium [30] would lead to endometrial hyperplasia and carcinoma in PCOS women (Fig. 3).
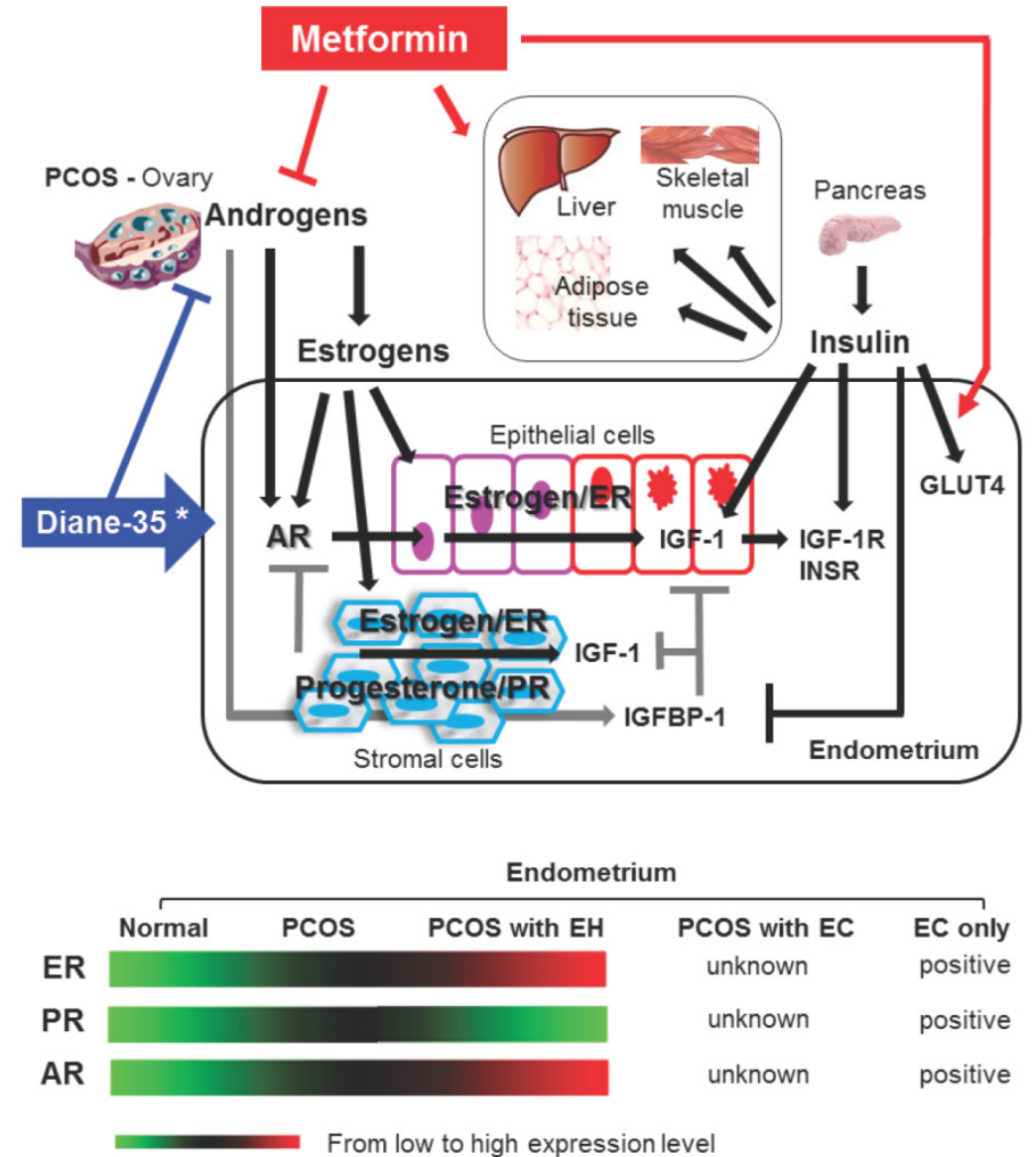

Figure 3. Schematic representation of hypothetical models and known mechanisms of Diane-35 and metformin actions in PCOS-IR women with early endometrial carcinoma. The proliferation and transformation of epithelial cells to carcinoma and the progression of endometrial cancer might account for a significant regulatory role of estrogen-dependent ER-mediated synthesis and activation of IGF-I signaling in PCOS women. Progesterone resistance is intimately related to PCOS-related endometrial pathology. Stromal PR-mediated IGFBP-I expression (gray lines) acts in an autocrine and paracrine manner to inhibit IGF-I signaling in both epithelial and stromal cells. Progesterone resistance results in a decrease in stromal IGFBP-I levels that in turn promotes IGF-I signaling in both epithelial and stromal cells. In addition, insulin might also promote epithelial cell proliferation and transformation to carcinoma in PCOS endometrium by blocking stromal IGFBP-I synthesis and consequently enhancing epithelial IGF-I levels and activities. IGF-I and insulin activate similar signaling pathways. The actions of Diane-35 (blue line) and metformin (red line) are indicated. * Cyproterone acetate, a key component of Diane-35, acts as an antagonist ligand for the AR and an agonist ligand for the PR. $\rightarrow$, stimulation or increase; $\dashv$, inhibition or decrease; AR, androgen receptor; ER, estrogen receptor; PR, progesterone receptor; IGF-I, insulin-like growth factor-I; IGF-IR, insulin-like growth factor-I receptor; IGFBP-I insulin-like growth factor binding protein-I; INSR, insulin receptor; GLUT4, glucose transporter 4; EH, endometrial hyperplasia; EC, endometrial carcinoma. 


\section{Towards a cure for early endometrial carcinoma in PCOS-IR women}

To the best of our knowledge, there are no available studies demonstrating the molecular mechanisms of combined treatment with Diane-35 and metformin in the endometrium. Although it is not clear whether the beneficial effects on endometrial carcinoma regression are due to improvement in hyperandrogenism, and/or insulin sensitivity, several possible explanations could account for this successful co-treatment (Fig. 3).

First, Diane-35 is composed of cyproterone acetate and ethinyl estradiol [16]. Although cyproterone acetate is a progesterone agonist, it also has anti-androgenic properties. Thus, increased progesterone action and attenuation of ovarian androgen production might affect the enhancement of PR-mediated IGFBP-1 activity and the inhibition of AR signaling in the endometrium. In addition, ethinyl estradiol might cause a suppression of ovarian estradiol production through inhibition of the hypothalamic-pituitary-ovarian axis [16]. As a result, overall endometrial ER-mediated IGF-1 expression and activity would decrease in PCOS women (Fig. 3).

Second, the most commonly described function of metformin is the modulation of insulin sensitivity and glucose metabolism in PCOS women [37]. Our patients had IR with compensatory hyperinsulinemia before treatment. Thus metformin might have stimulated insulin activity in the endometrium in addition to other insulin-target tissues such as liver, muscle, and adipose tissues (Fig. 3). However, the activation of insulin signaling is likely to promote the development of endometrial cancer [38]. In support of this view, our laboratory and others have shown that treatment with insulin increases IGF-1 levels and decreases IGFBP-1, IGF-1R, and insulin receptor levels in human endometrial stromal cells in vitro [31, 39-41]. Moreover, it is known that cancer cells have elevated glucose consumption and that insulin can stimulate glucose uptake by glucose transporters (GLUT). It has been shown that the levels of GLUT4 mRNA and protein are decreased in the endometrial cells from PCOS women compared to non-PCOS women $[42,43]$, and treatment with metformin increases GLUT4 mRNA and protein expression in endometrial cells from PCOS women [42].

Although there is still missing in vivo evidence for local insulin activity in the endometrial carcinoma from PCOS women, insulin-binding activity can be detected in human endometrial cancer cells [44] and insulin promotes endometrial cancer cell growth in vitro [45]. The interactions between endometrial and metabolic processes in PCOS-IR women remain to be characterized, but the results of the present study suggest alternative pathways for future investigations into the regulatory mechanisms of insulin in the initiation and development of endometrial carcinoma in PCOS-IR women. However, it should be noted that metformin also displays anti-cancer activities in a variety of other human cancers [46]. Previously, it has been shown that metformin inhibits cell proliferation and induces apoptosis of several endometrial cancer cell lines in a dose-dependent manner [47]. Recently, Sarfstein and colleagues have reported that treatment with metformin decreases cell proliferation and increases apoptosis in uterine serous carcinoma through inhibition of the insulin/IGF-I signaling pathway [48]. While progesterone resistance is associated with IR and decreased stromal PR activity [28], metformin is capable of reversing progesterone resistance and increasing PR expression in endometrial cancer cells [49, 50]. These data implicate the positive regulatory effects of metformin on the endometrial reversion from hyperplasia and/or carcinoma to normal endometria.

Epidemiological and clinical studies have provided ample evidence that endometrial cancer development is associated with being overweight or obese [11]. Weight loss in PCOS women through diet and/or exercise training has been shown to confer overall health benefits such as improvement of hyperandrogenism and insulin resistance, induction of ovulation, menstrual cyclicity $[3,51]$, and decreased plasma concentrations of circulating estrogen and IGF-1 [52]. In this report, all patients participated in the diet control and exercise training during treatment to increase IR and they experienced restored spontaneous ovulation that suppresses the long-term estrogen stimulation in the endometrium. Therefore, lifestyle modification might indirectly affect endometrial function and improve fertility.

Up to $75 \%$ of PCOS women are reported to be infertile [53]. The goal of combining Diane-35 and metformin for the treatment of early endometrial carcinoma in PCOS-IR women was to preserve the uterus for future pregnancy through the use of non-surgical methods. Our results suggest that the combined treatment led to rapid improvement in various endocrine and metabolic symptoms and promoted fertility in these women. Diet control and exercise training that might modulate the effect of the treatment with Diane-35 and metformin need to be considered. It is best to help patients obtain regulated ovulation and subsequent normal uterine pregnancy as early as possible after co-treatment.

\section{Limitations and reason for caution}

The number of patients in this study is low. This small trial size and the retrospective design of the 
study mean that the results presented here must be interpreted with caution. Future randomized trials with a larger sample size are needed to confirm our findings.

\section{CONCLUSIONS}

Our results show that this combined treatment could be a novel medical therapy for the treatment of early endometrial carcinoma in addition to reverse atypical endometrial hyperplasia in PCOS-IR women $[13,14]$. Further investigation of the molecular pathways in the endometrium of PCOS-IR women might reveal potential targets for this combined treatment to aid in the accurate and reproducible treatment in PCOS-IR women. These investigations will also provide additional insights into different responses of combined treatment in PCOS-IR women with early endometrial carcinoma.

\section{Acknowledgments}

This work was supported by the Shanghai Committee of Science and Technology, China (124119a4002), the Scientific Research Project of Shanghai Municipal Health Bureau, China (2013 4264), the Swedish Medical Research Council (5859 and 10380), Jane and Dan Olsson's Foundation, the Åke-Wiberg Foundation, and Clas Groschinsky's Foundation.

\section{Competing Interests}

The authors have declared that no competing interest exists.

\section{References}

1. Norman RJ, Dewailly D, Legro RS, Hickey TE. Polycystic ovary syndrome. Lancet 2007, 370:685-697.

2. Ehrmann DA. Polycystic ovary syndrome. N Engl J Med 2005, 352:1223-1236.

3. Moran LJ, Hutchison SK, Norman RJ, Teede HJ. Lifestyle changes in women with polycystic ovary syndrome. Cochrane Database Syst Rev 2011::CD007506.

4. Hardiman P, Pillay OC, Atiomo W. Polycystic ovary syndrome and endometrial carcinoma. Lancet 2003, 361:1810-1812.

5. Chittenden BG, Fullerton G, Maheshwari A, Bhattacharya S. Polycystic ovary syndrome and the risk of gynaecological cancer: a systematic review. Reprod Biomed Online 2009, 19:398-405.

6. Kim JJ, Kurita T, Bulun SE. Progesterone action in endometrial cancer, endometriosis, uterine fibroids, and breast cancer. Endocr Rev 2013, 34:130-162.

7. Lee WL, Lee FK, Su WH, Tsui KH, Kuo CD, Hsieh SL, Wang PH. Hormone therapy for younger patients with endometrial cancer. Taiwan J Obstet Gynecol 2012, 51:495-505.

8. Haoula Z, Salman M, Atiomo W. Evaluating the association between endometrial cancer and polycystic ovary syndrome. Hum Reprod 2012, 27:1327-1331.

9. Fearnley EJ, Marquart L, Spurdle AB, Weinstein P, Webb PM. Polycystic ovary syndrome increases the risk of endometrial cancer in women aged less than 50 years: an Australian case-control study. Cancer Causes Control 2010, 21:2303-2308.

10. Burzawa JK, Schmeler KM, Soliman PT, Meyer LA, Bevers MW, Pustilnik TL, Anderson ML, Ramondetta LM, Tortolero-Luna G, Urbauer DL, et al. Prospective evaluation of insulin resistance among endometrial cancer patients. Am J Obstet Gynecol 2011, 204:e351-357.

11. Kaaks R, Lukanova A, Kurzer MS. Obesity, endogenous hormones, and endometrial cancer risk: a synthetic review. Cancer Epidemiol Biomarkers Prev 2002, 11:1531-1543.
12. Tong $\mathrm{XM}$, Lin $\mathrm{XN}$, Jiang HF, Jiang LY, Zhang SY, Liang FB. Fertility-preserving treatment and pregnancy outcomes in the early stage of endometrial carcinoma. Chin Med J (Engl) 2013, 126:2965-2971.

13. Shen ZQ, Zhu HT, Lin JF. Reverse of progestin-resistant atypical endometrial hyperplasia by metformin and oral contraceptives. Obstet Gynecol 2008, 112:465-467.

14. Session DR, Kalli KR, Tummon IS, Damario MA, Dumesic DA. Treatment of atypical endometrial hyperplasia with an insulin-sensitizing agent. Gynecol Endocrinol 2003, 17:405-407.

15. Fauser BCJM. Revised 2003 consensus on diagnostic criteria and long-term health risks related to polycystic ovary syndrome (PCOS). Hum Reprod 2004, 19:41-47.

16. Jing Z, Liang-Zhi X, Tai-Xiang W, Ying T, Yu-Jian J. The effects of Diane-35 and metformin in treatment of polycystic ovary syndrome: an updated systematic review. Gynecol Endocrinol 2008, 24:590-600.

17. Odicino F, Pecorelli S, Zigliani L, Creasman WT. History of the FIGO cancer staging system. Int J Gynaecol Obstet 2008, 101:205-210.

18. Legro RS, Zaino RJ, Demers LM, Kunselman AR, Gnatuk CL, Williams NI, Dodson WC. The effects of metformin and rosiglitazone, alone and in combination, on the ovary and endometrium in polycystic ovary syndrome. Am J Obstet Gynecol 2007, 196:e401-410; discussion 402 e410-401.

19. Wheeler MJ. The determination of bio-available testosterone. Ann Clin Biochem 1995, 32 ( Pt 4):345-357.

20. Matthews DR, Hosker JP, Rudenski AS, Naylor BA, Treacher DF, Turner RC. Homeostasis model assessment: insulin resistance and beta-cell function from fasting plasma glucose and insulin concentrations in man. Diabetologia 1985, 28:412-419.

21. Blackburn GL, Bistrian BR, Maini BS, Schlamm HT, Smith MF. Nutritional and metabolic assessment of the hospitalized patient. JPEN J Parenter Enteral Nutr 1977, 1:11-22.

22. Shao R, Weijdegard B, Ljungstrom $K$, Friberg A, Zhu C, Wang X, Zhu Y, Fernandez-Rodriguez J, Egecioglu E, Rung E, Billig H. Nuclear progesterone receptor $A$ and $B$ isoforms in mouse fallopian tube and uterus: implications for expression, regulation, and cellular function. Am J Physiol Endocrinol Metab 2006, 291:E59-72.

23. Shang K, Jia X, Qiao J, Kang J, Guan Y. Endometrial abnormality in women with polycystic ovary syndrome. Reprod Sci 2012, 19:674-683.

24. Graham JD, Clarke CL. Physiological action of progesterone in target tissues. Endocr Rev 1997, 18:502-519.

25. Vrbikova J, Cibula D. Combined oral contraceptives in the treatment of polycystic ovary syndrome. Hum Reprod Update 2005, 11:277-291.

26. Rattanachaiyanont $M$, Angsuwathana $S$, Techatrisak $K$, Tanmahasamut $P$, Indhavivadhana S, Leerasiri P. Clinical and pathological responses of progestin therapy for non-atypical endometrial hyperplasia: a prospective study. J Obstet Gynaecol Res 2005, 31:98-106.

27. Jadoul P, Donnez J. Conservative treatment may be beneficial for young women with atypical endometrial hyperplasia or endometrial adenocarcinoma. Fertil Steril 2003, 80:1315-1324.

28. Aghajanova L, Velarde MC, Giudice LC. Altered gene expression profiling in endometrium: evidence for progesterone resistance. Semin Reprod Med 2010, 28:51-58.

29. Shao R, Wang X, Weijdegard B, Norstrom A, Fernandez-Rodriguez J, Brannstrom M, Billig $\mathrm{H}$. Coordinate regulation of heterogeneous nuclear ribonucleoprotein dynamics by steroid hormones in the human Fallopian tube and endometrium in vivo and in vitro. Am J Physiol Endocrinol Metab 2012, 302:E1269-1282.

30. Wang HS, Chard T. IGFs and IGF-binding proteins in the regulation of human ovarian and endometrial function. J Endocrinol 1999, 161:1-13.

31. Lin J, Li R, Zhou J. The influence of insulin on secretion of IGF-I and IGFBP-I in cultures of human endometrial stromal cells. Chin Med J (Engl) 2003, 116:301-304.

32. Fowler DJ, Nicolaides $\mathrm{KH}$, Miell JP. Insulin-like growth factor binding protein-1 (IGFBP-1): a multifunctional role in the human female reproductive tract. Hum Reprod Update 2000, 6:495-504.

33. McCampbell AS, Broaddus RR, Loose DS, Davies PJ. Overexpression of the insulin-like growth factor I receptor and activation of the AKT pathway in hyperplastic endometrium. Clin Cancer Res 2006, 12:6373-6378.

34. Fujimoto J, Nishigaki M, Hori M, Ichigo S, Itoh T, Tamaya T. Biological implications of estrogen and androgen effects on androgen receptor and its mRNA levels in human uterine endometrium. Gynecol Endocrinol 1995, 9:149-155.

35. Fujimoto J, Nishigaki M, Hori M, Ichigo S, Morishita S, Tamaya T. Effects of Estradiol and Testosterone on the Synthesis, Expression and Degradation of Androgen Receptor in Human Uterine Endometrial Fibroblasts. J Biomed Sci 1995, 2:160-165.

36. Slayden OD, Nayak NR, Burton KA, Chwalisz K, Cameron ST, Critchley HO, Baird DT, Brenner RM. Progesterone antagonists increase androgen receptor expression in the rhesus macaque and human endometrium. J Clin Endocrinol Metab 2001, 86:2668-2679.

37. Palomba S, Falbo A, Zullo F, Orio F, Jr.. Evidence-based and potential benefits of metformin in the polycystic ovary syndrome: a comprehensive review. Endocr Rev 2009, 30:1-50.

38. Djiogue S, Nwabo Kamdje AH, Vecchio L, Kipanyula MJ, Farahna M, Aldebasi Y, Seke Etet PF. Insulin resistance and cancer: the role of insulin and IGFs. Endocr Relat Cancer 2013, 20:R1-R17. 
39. Capp E, Jauckus J, von Eye Corleta H, Toth B, Strowitzki T, Germeyer A. Does metformin influence the insulin-, IGF I- and IGF II-receptor gene expression and Akt phosphorylation in human decidualized endometrial stromal cells? Eur J Obstet Gynecol Reprod Biol 2011, 158:248-253.

40. Germeyer A, Jauckus J, Zorn M, Toth B, Capp E, Strowitzki T. Metformin modulates IL-8, IL-1beta, ICAM and IGFBP-1 expression in human endometrial stromal cells. Reprod Biomed Online 2011, 22:327-334.

41. Giudice LC, Dsupin BA, Irwin JC. Steroid and peptide regulation of insulin-like growth factor-binding proteins secreted by human endometrial stromal cells is dependent on stromal differentiation. J Clin Endocrinol Metab 1992, 75:1235-1241.

42. Zhai J, Liu CX, Tian ZR, Jiang QH, Sun YP. Effects of metformin on the expression of GLUT4 in endometrium of obese women with polycystic ovary syndrome. Biol Reprod 2012, 87:29.

43. Mioni R, Chiarelli S, Xamin N, Zuliani L, Granzotto M, Mozzanega B, Maffei P, Martini C, Blandamura S, Sicolo N, Vettor R. Evidence for the presence of glucose transporter 4 in the endometrium and its regulation in polycystic ovary syndrome patients. J Clin Endocrinol Metab 2004, 89:4089-4096.

44. Nagamani M, Stuart CA. Specific binding and growth-promoting activity of insulin in endometrial cancer cells in culture. Am J Obstet Gynecol 1998, 179:6-12.

45. Zhang G, Li X, Zhang L, Zhao L, Jiang J, Wang J, Wei L. The expression and role of hybrid insulin/insulin-like growth factor receptor type 1 in endometrial carcinoma cells. Cancer Genet Cytogenet 2010, 200:140-148.

46. Martin-Castillo B, Vazquez-Martin A, Oliveras-Ferraros C, Menendez JA. Metformin and cancer: doses, mechanisms and the dandelion and hormetic phenomena. Cell Cycle 2010, 9:1057-1064.

47. Cantrell LA, Zhou C, Mendivil A, Malloy KM, Gehrig PA, Bae-Jump VL. Metformin is a potent inhibitor of endometrial cancer cell proliferation--implications for a novel treatment strategy. Gynecol Oncol 2010, 116:92-98.

48. Sarfstein R, Friedman Y, Attias-Geva Z, Fishman A, Bruchim I, Werner H. Metformin downregulates the insulin/IGF-I signaling pathway and inhibits different uterine serous carcinoma (USC) cells proliferation and migration in p53-dependent or -independent manners. PLoS One 2013, 8:e61537.

49. Xie Y, Wang YL, Yu L, Hu Q, Ji L, Zhang Y, Liao QP. Metformin promotes progesterone receptor expression via inhibition of mammalian target of rapamycin (mTOR) in endometrial cancer cells. J Steroid Biochem Mol Biol 2011, 126:113-120.

50. Dong L, Zhou Q, Zhang Z, Zhu Y, Duan T, Feng Y. Metformin sensitizes endometrial cancer cells to chemotherapy by repressing glyoxalase I expression. J Obstet Gynaecol Res 2012, 38:1077-1085.

51. Thomson RL, Buckley JD, Brinkworth GD. Exercise for the treatment and management of overweight women with polycystic ovary syndrome: a review of the literature. Obes Rev 2011, 12:e202-210.

52. Carlson MJ, Thiel KW, Yang S, Leslie KK. Catch it before it kills: progesterone, obesity, and the prevention of endometrial cancer. Discov Med 2012, 14:215-222.

53. Homburg R. Management of infertility and prevention of ovarian hyperstimulation in women with polycystic ovary syndrome. Best Pract Res Clin Obstet Gynaecol 2004, 18:773-788. 